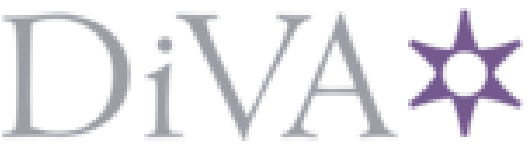

http://www.diva-portal.org

Postprint

This is the accepted version of a paper presented at IEEE - 13th System of Systems Engineering Conference.

Citation for the original published paper:

Jinzhi, L., Chen, D., Jian, W., Törngren, M. (2018)

Towards A Service-oriented Framework for MBSE Tool-chain Development

In:

N.B. When citing this work, cite the original published paper.

Permanent link to this version:

http://urn.kb.se/resolve?urn=urn:nbn:se:kth:diva-226673 


\title{
Towards A Service-oriented Framework for MBSE Tool-chain Development
}

\author{
Jinzhi Lu*, DeJiu Chen*, Jian Wang ${ }^{\dagger}$, Martin Torngren* \\ * KTH Royal Institute of Technology, 100 44, Stockholm \\ $\dagger$ University of Electronic Science and Technology, 611731, China \\ Email: jinzhl@kth.se
}

\begin{abstract}
This paper proposes a SPIRIT framework supporting model-based systems engineering (MBSE) tool-chain development of advanced cyber-physical systems (CPS) with emphasis on tool integration, process management, automated verification and validation. The core features of the developed MBSE tool-chain include domain-specific modeling to describe CPS development, service-oriented deployment of technical resources (data, model and tool operations) and process management through IT platforms. The framework has two purposes: to support tool-chain development with a systems engineering approach; to promote interoperability of the whole developed tool-chain through a service-oriented approach. The framework covers social, process, information and technical aspects aiming to integrate various related MBSE techniques with tool-chain development. Based on the framework, an MBSE tool-chain prototype is developed, and the flexibility and interoperability are evaluated through a case study.
\end{abstract}

Keywords: MBSE; Tool-chain development; Process management; Tool integration; Service orchestration

\section{INTRODUCTION}

Model-based Systems Engineering (MBSE) is proposed to deal with the increasing complexity of CPS. However, current MBSE tools are focused on specific domains, developed based on isolated and unrelated modeling theories and for specific purposes with different semantic models and uncommon storage representations - all of which challenge seamless integration between tools [1]. Moreover, heterogeneous and insufficient APIs provided by tools make platform, presentation and control integrations difficult across the whole life cycle [2]. Given the current status of MBSE, one of the challenges is integration across disciplines during the whole life cycle [3]. MBSE tool-chains are considered as a good solution to deal with this integration [4], and views of platform, presentation, data, control and process need to be considered during development [5].

In addition to the integration issues, MBSE tool-chain development is also challenged by domain-specific views, process management and automated verification $\&$ validation $(\mathrm{V} \& \mathrm{~V})$. Since CPS consists of multi-domain systems crossdomain software and hardware [6], focusing on different composite systems (their system characteristics), there is need to formalize them based on models using related domainspecific views. Moreover, the growing complexity means that development processes are no longer flexible, efficient and agile [7]. Particularly during a co-design and collaborative process, deployment of technical resources related to MBSE are much more complex than before. In addition, automated $\mathrm{V} \& \mathrm{~V}$, configuration and change management need to be integrated with process management in order to promote efficiency of CPS development.

In response to these challenges, we propose a serviceoriented framework called SPIRIT to support MBSE toolchain development. It consists of four layers( Social, Process, Information \& seRvice Infrastructure (IRI) and Technical) and a learning-before-doing approach based on systems engineering. In addition, we adopt a service-oriented technique to integrate domain-specific modeling with process management, deployment of technical resources and automated verification and validation to for better interoperability.

In this paper, we present our solution in detail. We describe related work in Section II and the SPIRIT framework in Section III. Section IV introduces an MBSE tool-chain prototype developed based on the SPIRIT framework. We discuss the case study in Section $\mathrm{V}$ and offer our conclusions in Section VI.

\section{RELATED WORK}

\section{A. Domain-specific modeling}

Domain-specific modeling (DSM) is an engineering approach to describe facets of system abstractions through DSM models built based on domain-specified modeling language (DSML) to describe information for specific domains and systems through a set of formal meta-models [8], e.g., EASTADL [9], VHDL [10]. Developers make use of such metamodels to create DSM models to formalize related information about CPS products and their development.

\section{B. Tool integration}

Tool-integration refers to activities that produce an integrated environment supporting the development process of CPS by sharing and reusing artifacts generated from different tools, tracing between such artifacts and accessing them from different stakeholders across the whole life cycle [11]. As summarized from existing solutions from the technical view, four types of tool integration are shown as follows: 


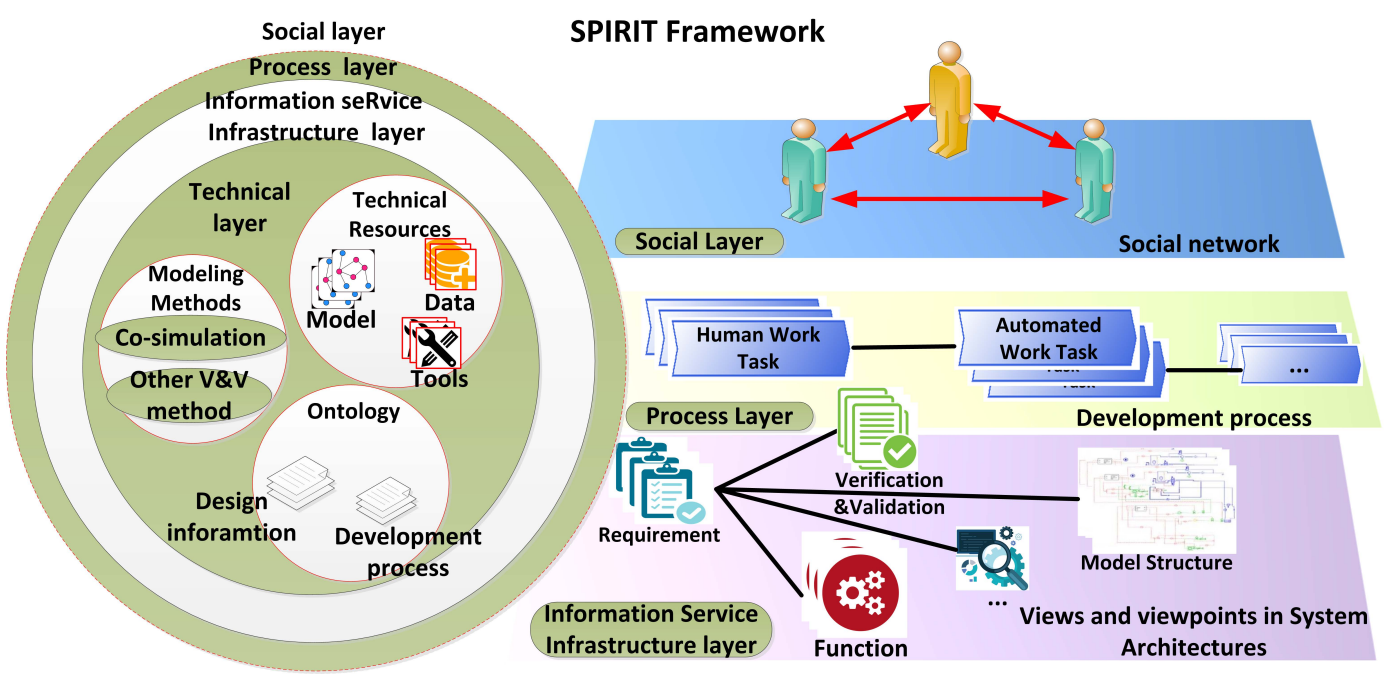

Fig. 1. SPIRIT Framework

- Linked data is used to describe a recommended best practice for exposing, sharing and connecting data, information, and knowledge on semantic webs using URIs and RDF, such as OSLC [12].

- Neutral data exchange is a standard-based approach for information exchange in favour of both the tool suppliers and the organizations that use multiple design tools within a whole life cycle, such as STEP AP233 [13].

- Meta-model integration refers to an approach facilitating model and data integration using transformations of metamodel and meta-data between tools such as [14].

- Tool-based integration refers to a specific tool providing related services enabling integration of models and APIs of tools such as ModelBus [15].

\section{Process management based on IT platforms}

Business process management (BPM) refers to a discipline to discover, model, analyze, improve and automate business processes using IT platforms [16]. Currently, there are several options to support business process modeling, e.g. activity diagram in UML [17], BPMN [18]. After the business process is formalized by such models, process engines can be implemented to generate related management systems for process control and monitoring, such as BPM Camunda [19].

In this paper, we concentrate on a framework for MBSE tool-chain development. First, the framework captures the toolchain developers' requirements for the target MBSE toolchain, based on a systems engineering approach. Second, the framework provides a service-oriented approach to develop concepts.

\section{SERVICE-ORIENTEd FrAMEWORK SUPPORTING MBSE TOOL-CHAIN DEVELOPMENT}

\section{A. SPIRIT Framework}

In order to support MBSE tool-chain development, the SPIRIT framework is proposed to capture requirements using a systems engineering approach. Based on a SPIT (Social, Process, Information and Technical layers) framework [4] in which one MBSE tool-chain is considered as one system whose architecture description includes viewpoints capturing related stakeholders' concerns. In contrast to SPIT, SPIRIT makes use of an IRI layer to replace the Information layer shown as Fig. 1. We summarize the concepts in each layer as follows:

The Social layer involves an explicit network of the different stakeholders (and the relationships between them) using the MBSE tool-chain. The stakeholders are related to the development processes implemented by the MBSE tool-chain, and address views in architecture descriptions of their target products. They also implement their own tasks and access the corresponding technical resources (models, data and tools). The implicit factors in the social layer are the rulers and policies of such social networks, the concerns of tool-chain developers and the environment of the related tool-chains.

In the Process layer, development processes related to the target products are considered. Each development process consists of Work Task concepts including Human Work Task and Automated Work Task, and Gate concepts representing decision making of the work flows and relationships between them.

In the IRI layer, views covered by the system architecture descriptions of related products are considered, such as requirement, model structure and function, etc. They represent the information of system artifacts in each Work Task (e.g. what's the requirement?...) and deployment of related technical resources. Moreover, such information and technical resources are wrapped as services in a service infrastructure which can be accessed through URL (Introduced in Section C).

The $\boldsymbol{T}$ echnical layer covers ontology, modeling methods and technical resources. Ontology refers to explicit formal standard instructions about development processes in the Process layer and information of system artifacts (system views) in the IRI 


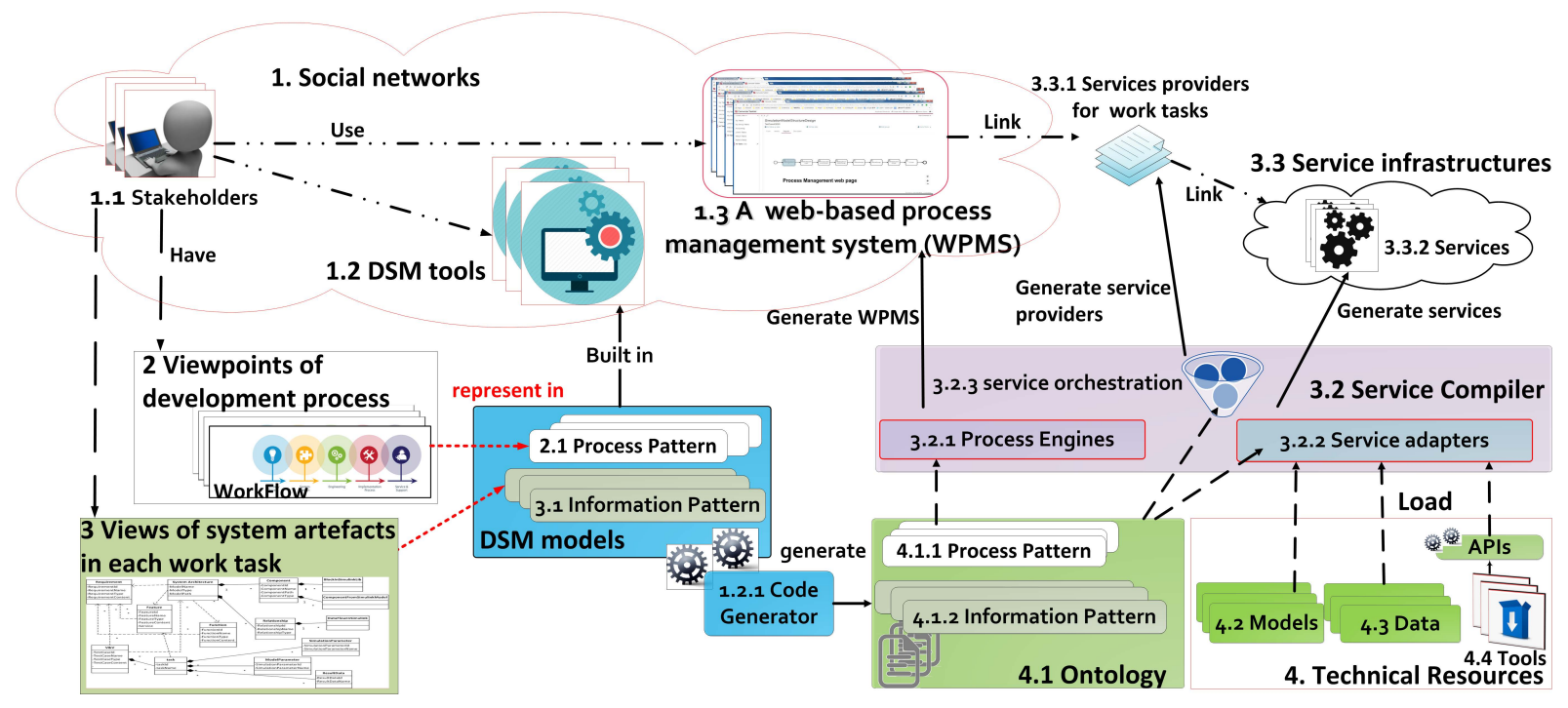

Fig. 2. MBSE Tool-chain Workflows Supported by SPIRIT

layer. Modeling methods (how to model) are considered based on development process and related views, e.g. simulation in Matlab/Simulink is used for verification \& validation $(\mathrm{V} \& \mathrm{~V})$ (introduced in Section IV). The technical resources include data, model, codes and tool APIs supporting development process and system views and enabling modeling methods.

\section{B. Tool-chain Concepts Developed based on SPIRIT}

After capturing the requirements of the MBSE tool-chain, a concept is proposed to integrate domain-specific views, tool-integration and process management based on a service infrastructure. In Fig.2, the workflow of the tool-chain concept is proposed:

(1) In the social networks, stakeholders who work in the development process and have related views build DSM models using related tools. Therefore, the social network (red boxes) includes:

- Stakeholders participating in the product development (Part 1.1).

- DSM tools used to build models to represent the development processes and information (system views) of system artifacts (Part 1.2).

- Web-based process management system (WPMS) embedded with services of information and technical resource deployment for stakeholders to implement their work tasks (Part 1.3).

(2) Development processes are formalized by the process pattern (Part 2.1) of DSM models.

(3) System views related to development processes are formalized by the information pattern (Part 2.2) of DSM models, such as model structures, V\&V.

(4) Through a developed code generator, DSM models are transformed to ontology in XML (Part 4.1) including process pattern (Part 4.1.1) and information pattern (Part 4.1.2).
(5) Technical resources are used to support CPS development as follows:

- Models (Part 4.1) supporting work tasks, e.g. Simulink models.

- Data (Part 4.2) used for works tasks.

- Tools (Part 4.3) used for work tasks, in particular, providing APIs.

(6) A service compiler is used to generate a WPMS with related services of technical resources and system artifact information.

- A process engine (Part 3.2.1) loads process pattern to generate a WPMS with related work tasks.

- Service adapters (3.2.2) generate the corresponding services of information of system artifacts and deployment of the related technical resources.

- A service orchestration (3.2.3) loads ontology to manage, configure and orchestrate services to the related providers for work tasks in the development process (introduced in Part D).

(7) A service infrastructure (3.3) refers to a collection of services accessing related information and deployment of technical resources.

(8) The service orchestration can generate service providers linked to the related work tasks in the WPMS. Moreover, such service providers also link the required services of information and technical resources in the service infrastructure.

(9) Finally, stakeholders implement related development processes based on WPMS.

\section{DSM Supporting MBSE tool-chain}

In order to formalize the development processes and system views, stakeholders first design meta-models based on metameta models in DSM tools. For example, MetaEdit+ provides a GOPPR approach with formal meta-meta model concepts 


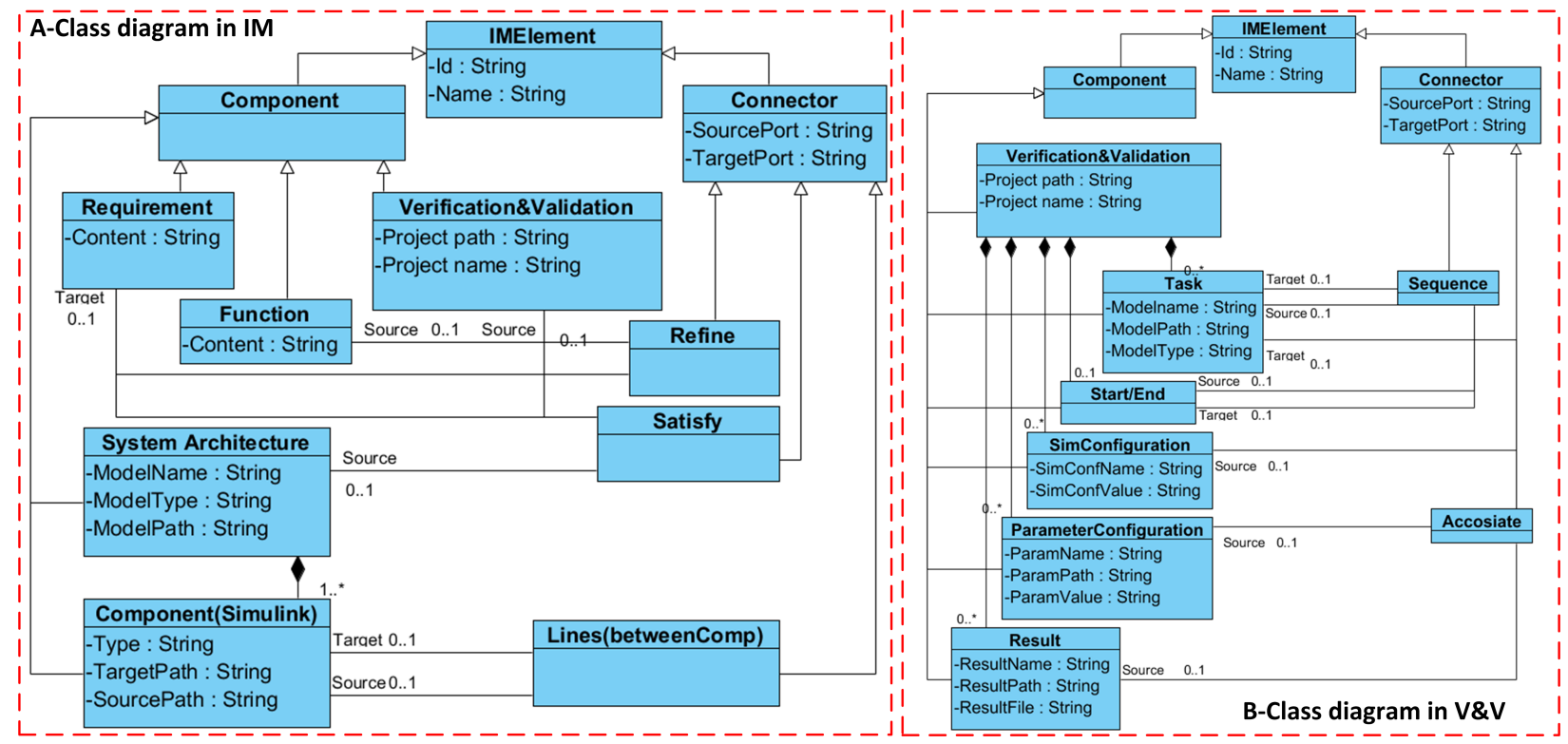

Fig. 3. A-Class diagram of Information Pattern in Each Work Task and B- Class diagram of Verification\&Validation

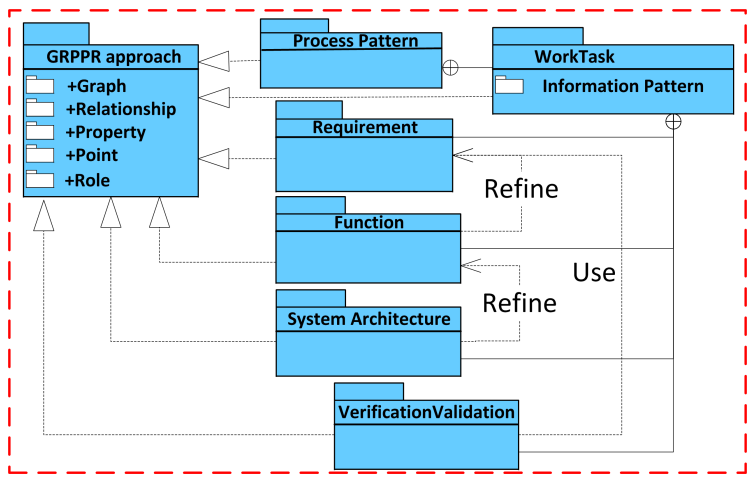

Fig. 4. Package Dependencies

to define and develop meta-models [20]. Then based on such meta-models, DSM models are built to represent development processes and related information and are used to generate ontologies.

The DSM models include process patterns and information patterns. As shown in Fig.2, the process patterns represent CPS development processes and the information patterns describe information of system artifacts (system views) in each work task. We propose several definitions to support meta-model development. Fig.4 represents the packages of requirement, function, system architecture and V\&V. In this paper, we adapt the GOPPR approach to develop the initial meta-models ${ }^{1}$.

Definition 1: A process pattern $a$ refers to a Graph $^{2}, P M_{a}$ :

\footnotetext{
${ }^{1}$ The meta-modeling language, an extension of GOPPR is introduced in http://users.jyu.fi/ jpt/ME2000/Me07/sld003.html.

${ }^{2}$ Graph refers to a collection of object, relationship and role types represented as one window (one integrated concept of class diagram and package in UML).
}

$$
\begin{aligned}
& P M_{a}=\operatorname{Set}(\text { Set }(\text { WorkTask_Human }, i \geq 0), \\
& \text { Set }\left(\text { WorkTask_Auto }_{n}, n \geq 0\right), \operatorname{Set}\left(\text { Seq }_{m}, m \geq 0\right), \\
& \text { Set }_{\left.\left(\text {Gate }_{k}, k \geq 0\right), \text { start }, \text { end }\right)}
\end{aligned}
$$

where Set() refers to one collection; The Object $^{3}$ WorkTask_Human $_{i}$ refers to one work task node representing one work task $i$ that stakeholders need to participate in; The Object WorkTask_Auton refers to one work task node representing a work task $n$ which a computer does automatically; Each Relationship ${ }^{4} \mathrm{Seq}_{m}$ is one edge between nodes which represents sequence flow $m$; Object start and end represent one start node and one end node,

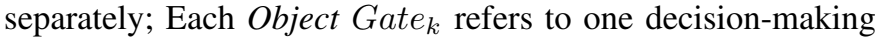
node $k$ based on Gateway in BPMN [18].

Each information pattern formalizes system views of product architecture related to the corresponding work task, such as Objects requirement, function, model structure and $\mathrm{V} \& \mathrm{~V}$ (The class diagram of the information pattern as one example is shown in Fig.3-A and B). The Objects model structure, function and $\mathrm{V} \& \mathrm{~V}$ associated with the Object requirement. The Object model structure represents a simulation model structure. It includes Objects component and line representing blocks in the model and links between them. For example, when an Object model structure represents a Simulink model, its components and relationships represent blocks and lines in the model, if Matlab/Simulink is selected as a simulation tool for V\&V. Object function describes functions of a simulation model and related components. The Object V\&V associated with requirement contains Objects task, start, end, SimulationParameter, ModelParameter and ResultData to describe $\mathrm{V} \& \mathrm{~V}$ activities. In $\mathrm{V} \& \mathrm{~V}$, each Object task is defined as one

\footnotetext{
${ }^{3}$ Object refers to describe a thing with properties, relationships and roles, such as class in UML.

${ }^{4}$ Relationship refers to one link between two Objects.
} 
simulation execution with specific configurations according to the corresponding Objects.

Definition 2: The information pattern related to one work task $(i \| n)$ in the process $a$ is defined as the Graph $I M_{(a, i)}$.

$$
\begin{aligned}
& I_{(a, i \| n)}=\text { WorkTaskDecomposite }( \\
& \text { WorkTask_Human } \left.{ }_{i} \| \text { WorkTask_Auto }{ }_{n}\right)
\end{aligned}
$$

$$
\begin{aligned}
& I M=\operatorname{Set}\left(\operatorname{Set}\left(\operatorname{Req}_{n}, n \geq 0\right), \operatorname{Set}\left(F_{u n c_{m}}, m \geq 0\right),\right. \\
& \operatorname{Set}\left(\operatorname{sArc}_{k}, k \geq 0\right), \operatorname{Set}\left(V \& V_{x}, x \geq 0\right), \\
& \operatorname{Set}(I M \text { Relationship }, y \geq 0))
\end{aligned}
$$

where WorkTaskDecomposite() refers to a decomposition that one Object WorkTask (WorkTask_Human and WorkTask_Auto) decomposed into a new Graph $I M_{(a, i)}$; $R e q_{n}$ is a Object requirement $n ; F u n c_{m}$ refers to one Object function $m$; Object $s A r c_{k}$ refers to a model structure $k . V \& V_{x}$ refers to one Object $\mathrm{V} \& \mathrm{~V} x$. IMRelationship $y$ refers to relationships between previous Objects in IM.

$$
\begin{aligned}
& \operatorname{sArc}_{k}=\left(\operatorname{Set}\left(\operatorname{Comp}_{n}, n \geq 0\right),\right. \\
& \left.\operatorname{Set}\left(\operatorname{CompRel}_{m}, m \geq 0\right)\right)
\end{aligned}
$$

where $\mathrm{Comp}_{n}$ and CompRel $m$ refer to the component $n$ and line $m$ between components in the model structure.

$$
\begin{aligned}
& V \& V=\left(\text { Set }_{\left(\text {task }_{n}, n \geq 0\right),},\right. \\
& \operatorname{Set}\left(\text { RelationshipsInVV }_{m}, m \geq 0\right), \text { start }, \text { end, } \\
& \operatorname{Set}\left(S M C_{a}, a \geq 0\right), \operatorname{Set}\left(\operatorname{SParam}_{b}, b \geq 0\right), \\
& \left.\operatorname{Set}\left(\operatorname{SRes}_{c}, c \geq 0\right)\right)
\end{aligned}
$$

where $\operatorname{task}_{n}$ refers to one node representing one simulation with given configurations defined based on $S M C_{a}$,

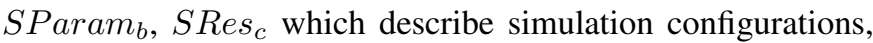
parameter configurations and simulation results, separately. RelationshipsInV $V_{m}$ refers to sequence and association relationships between nodes.

Definition 3: In the MBSE tool-chain, DSM models are transformed to services which can control tools and deploy technical resources, such as models. One simulation model related to $s A r c_{k}$ is defined by $S M\left(s A r c_{k}\right)$. Each component and relationship in the $S M\left(s A r c_{k}\right)$ are defined as $S M C \operatorname{Com}\left(\operatorname{Comp}_{n}\right)$ and $S M C R e l a\left(C_{\text {ompRel }}\right)$, separately; During V\&V, one simulation execution task $k_{n}$ is defined by $S R u n\left(\operatorname{task}_{n}\right)$; The simulation configuration $a$ for $\operatorname{SRun}\left(\operatorname{tas}_{n}\right)$ is defined by $\operatorname{SCon}\left(\operatorname{SRun}\left(\operatorname{tas}_{n}\right), a\right)$; The simulation parameter configuration $b$ for $\operatorname{SRun}\left(\operatorname{task}_{n}\right)$ is defined by $S$ Param $\left(S R u n\left(\operatorname{task}_{n}\right), b\right)$. The related simulation result $c$ is defined by $S R e s\left(S R u n\left(\operatorname{tas}_{n}\right), c\right)$; A mapping between DSM models and technical resources is defined as follows:

$$
a=\operatorname{ResMapping}(b)
$$

where ResMapping() refers to a mapping from one DSM model concept $\left(b=s A r c_{k} \ldots S R e s_{c}\right)$ or the related ontology concepts in DSM models to one technical resource $a \quad\left(a=S M\left(s \operatorname{Arc}_{k}\right) \ldots S \operatorname{Res}\left(\operatorname{SRun}\left(\operatorname{task}_{n}\right), c\right)\right)$. For example, $S M\left(s A r c_{k}\right)=\operatorname{ResMapping}\left(s A r c_{k}\right)$ where ResMapping() can be implemented by the property modelName as a tag to describe the real $S M$ it represents.
Definition 4: One tool operation is defined as an execution $\alpha$ of related tools to deploy the previous technical resources and support configurations:

$$
\begin{aligned}
& \text { toolOper }(\alpha, \text { tool }, \text { techResource, constraint, } \\
& \text { APItemplate, mode })
\end{aligned}
$$

where tool refers to the related tool supporting such tool operations. techResource refers to technical resources used for such tool operations, such as $s A r c_{k}$. constraint refers to constraints of the tool operations, e.g. during creating one model, one block from the original model should be copied to a target path which the original block and the target path refer to as the techResource and constraint, separately. APItemplate refers to a set of APIs supporting the related operations; mode refers to a trigger of the related tool operation, such as executing manually or automatically.

\section{SPIRIT Framework Supporting Tool integration}

As shown in Fig.2, after DSM models are built, ontology is generated through a developed code generator. Then the related concepts in ontologies are transformed to a WPMS and related services of deployment of technical resources and information of system artifacts. Such services, constructing a service infrastructure, wrap technical resources and ontology into unified forms which can be accessed through URL. Moreover, a service orchestration is developed to link related work tasks in the WPMS and required services of technical resources and information in ontologies. The related concepts are defined as follows:

Definition 5: An ontology concept, $O n g(x)$ refers to one concept mirrored from the $x$ concept in $P M$ and $I M$.

Definition 6: A service concept, $\operatorname{Ser}_{n}(x)$ is a web service $n$ offered by service adapters for ontology and technical resources, which $x$ refers to $O n g(x)$ and technical resources, such as $S M$ and toolOper.

Definition 7: A service provider $i$ for one work task, $\operatorname{SerProvider}_{i}=\operatorname{Set}\left(\operatorname{Ser}_{n}(x), n \geq 0\right)$ is a set of services in each work task $i$.

Definition 8: $w$ Process $_{a}$ refers to one process $a$ in WPMS. $w_{\text {Task_Human }}^{(a, i)}$ and wTask_Auto $_{(a, n)}$ refer to work task $i$ for human and work task $n$ implementing automatically in $w$ Process $s_{a}$.

Definition 9: Notation $a->b$ refers to one transformation between $a$ and $b$ which represent concepts in DSM models, ontologies, services and WPMS. Notation $a \mid>b$ refers to one link from $a$ to $b$ which represent concepts in services, service providers and WPMS.

In Algorithm 1, an approach is presented to generate wProcess $_{a}$ from DSM models. Firstly, DSM models including $P M_{a}$ and $I M_{(a, i)}$ are transformed to the corresponding ontology concepts using a developed code-generator. Then the ontology concepts related to $O n g\left(P M_{a}\right)$ are used to generate a web-based process management system through a developed compiler. The each $\operatorname{Ong}\left(I M_{(a, i)}\right)$ is transformed to the related SerProvider $i$ linked with wTask_Human $_{(a, i)}$ in the Process $_{a}$. Moreover, based on 


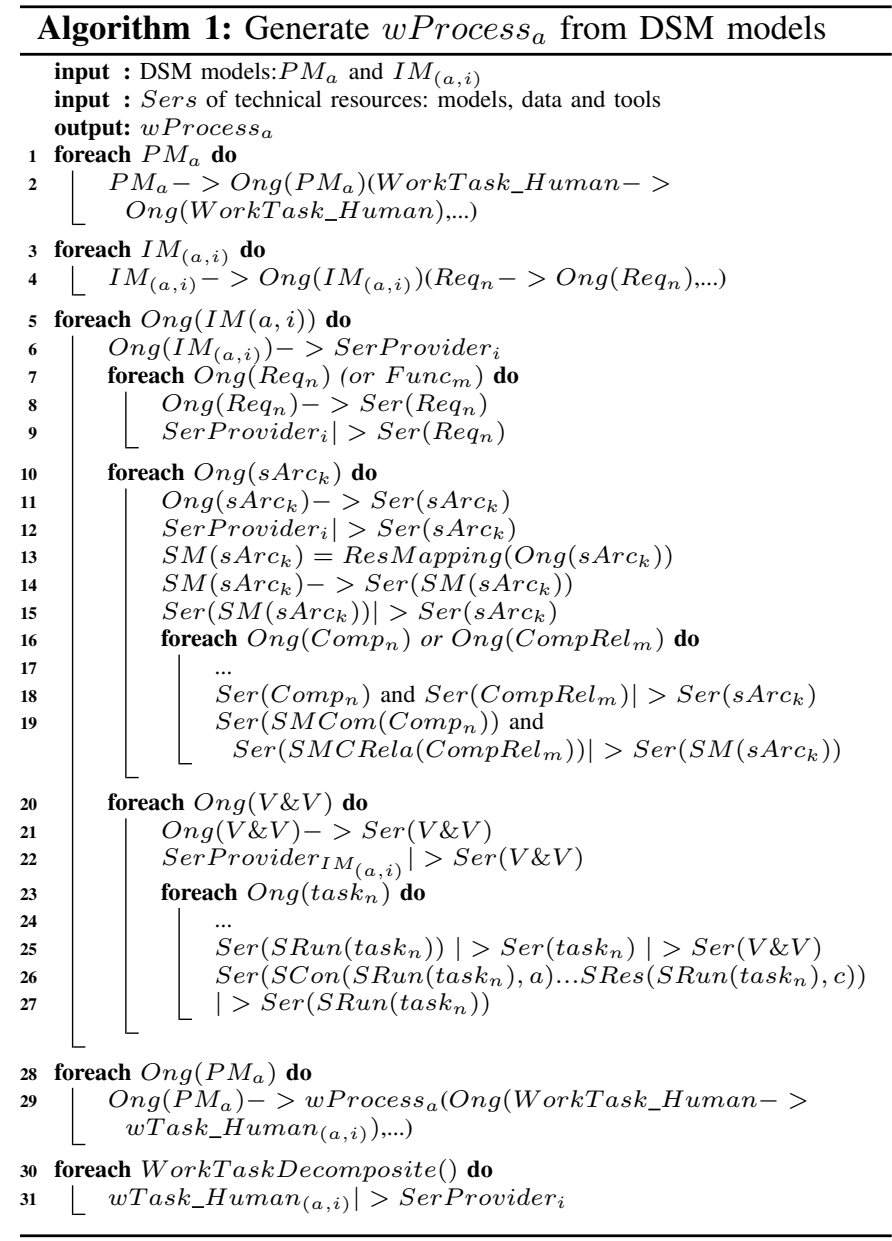

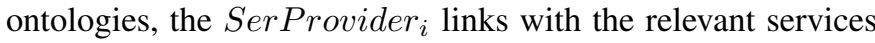
of $\operatorname{Ong}\left(I M_{(a, i)}\right)$ in each work task.

TABLE I

SERVICES OF TOOL OPERATIONS SUPPORTING TECHNICAL RESOURCES

\begin{tabular}{|c|c|c|}
\hline $\begin{array}{l}\text { Service Concepts of } \\
\text { Technical resoruces }\end{array}$ & Tool operation service & Purposes of tool operations \\
\hline $\operatorname{Ser}\left(S M\left(s A r c_{k}\right)\right)$ & $\begin{array}{l}\text { Sers to open/close tools } \\
\text { Sers to open/close/create... models }\end{array}$ & Add/Delete/Change/Query models \\
\hline $\operatorname{Ser}\left(\operatorname{SMCom}\left(\mathrm{Comp}_{n}\right)\right)$ & Sers to add/delete/... blocks & Add/Delete/Change/Query blocks \\
\hline $\operatorname{Ser}\left(\right.$ SMCRela $\left(\right.$ CompRelm $\left.\left._{m}\right)\right)$ & Sers to add/delete/... lines & Add/Delete/Change/Query lines \\
\hline $\operatorname{Ser}\left(\operatorname{SRun}\left(\operatorname{task}_{n}\right)\right)$ & Sers to execute simulations & Execute one simulation \\
\hline $\operatorname{Ser}\left(\operatorname{SCon}\left(\operatorname{SRun}\left(\operatorname{task}_{n}\right), a\right)\right.$ & Sers to configure solvers & Configure solvers \\
\hline Ser $\left(\operatorname{SParam}\left(\right.\right.$ SRun task $\left.\left.\left._{n}\right), b\right)\right)$ & Sers to configure parameters & Configure parameters \\
\hline $\operatorname{Ser}\left(\operatorname{SRes}\left(\operatorname{SRun}_{\left.\left.\left(\operatorname{task}_{n}\right), c\right)\right)}\right.\right.$ & Sers to browse the results & Browse results on the webs \\
\hline
\end{tabular}

In addition, services of technical resources are linked with the corresponding services of $\operatorname{Ong}\left(I M_{(a, i)}\right)$, such as $\operatorname{Ser}\left(S M\left(s A r c_{k}\right)\right)$ and $\operatorname{Ser}\left(\operatorname{SRun}\left(\operatorname{task}_{n}\right)\right)$ are linked with $\operatorname{Ser}\left(s A r c_{k}\right)$ and $\operatorname{Ser}\left(\operatorname{tas}_{n}\right)$, separately. As shown in TABLE $\mathrm{I}$, in order to implement tool operations to support deployment of technical resources, each service of technical resources is linked with the related tool operation services. Finally, stakeholders can access the technical resources and implement related tool operations through such services.

\section{Case Study}

Based on the SPIRIT framework, we developed a toolchain prototype using the tools shown in Table II. The related
TABLE II

TOOLS USED IN THE TOOL-CHAIN PROTOTYPE

\begin{tabular}{cc}
\hline Techniques & Tools \\
\hline Domain-specific modeling & MetaEdit+ \\
Ontology description & XML \\
Process modeling engine & BPM Camunda \\
Service-based infrastructure & Related techniques of OSLC \\
V\&V & Matlab/Simulink \\
\hline
\end{tabular}

workflow is shown in Fig.5:

(1) The meta-models developed in MetaEdit+. are used to build DSM models to formalizing the domain-specific views of CPS development. Then through a developed code generator, ontologies in XML file are generated.

(2) XML is loaded by a developed compiler including a process modeling engine, several OSLC adapters and a service orchestration.

(3) A WPMS is generated by the developed compiler.

(4) Stakeholders use their personal work tasks in the WPMS to implement their modeling work through related OSLC services.

The case study also demonstrates a process to build a Simulink model and execute one simulation by the tool-chain. Two main work tasks are as follows:

- Worktask1 refers to one work task to create a model with a Sin source block connecting to a Scope block in Simulink.

- Worktask2 refers to verification\&validation work task for model configurations and parameter settings, simulation executions and browsing the simulation result on one stakeholder's personal page in the WPMS.

When the WPMS is generated, each wTask_Human ${ }_{(a, i)}$ is linked with the related SerProvider ${ }_{i}$. In detail, in $\quad$ Task_Human $(a, 1) \quad$ (Worktask1), $\operatorname{Ser}\left(\operatorname{Req}_{1}\right)$, $\operatorname{Ser}\left(\right.$ Func $\left._{1}\right), \operatorname{Ser}\left(\right.$ sArc $\left._{1}\right)$ are linked through SerProvider . $_{1}$. The $\operatorname{Ser}\left(s A r c_{1}\right)$ are linked with $\operatorname{Ser}_{\left(S M\left(s A r c_{1}\right)\right) \text {. }}$. Moreover, $\operatorname{Ser}\left(C_{\text {Comp }}\right.$ sin $), \quad \operatorname{Ser}\left(\right.$ Comp $\left._{\text {scope }}\right)$ and $\operatorname{Ser}\left(\right.$ CompRel line $\left._{\text {lin }}\right)$ in $\operatorname{Ser}\left(s A r c_{1}\right)$ are linked with $\operatorname{Ser}\left(S M \operatorname{Com}\left(\operatorname{Comp}_{\text {sin }}\right)\right), \operatorname{Ser}\left(\operatorname{SMCom}\left(\operatorname{Comp}_{\text {scope }}\right)\right)$ and $\operatorname{Ser}\left(S M C R e l a\left(C_{\text {ompRel }}\right.\right.$ line $\left.)\right)$, separately. Stakeholders can access the related technical resources through their own URL. They can implement related tool operations to create models, such as 'create_model' and 'add_block' etc. In wTask_Human $(a, 2)$ (Worktask2), one developer can configure the Simulink model and execute the simulations through the related OSLC services. He/she can also browse the related simulation results in the WPMS.

\section{Discussion}

From the case study, we find the MBSE tool-chain prototype can support Matlab/Simulink executing simulations automatically. The stakeholders' domain-specific views are described by the DSM models built based on meta-models. Then the DSM models are transformed to a WPMS which controls Matlab/Simulink and deploys related models automatically. 


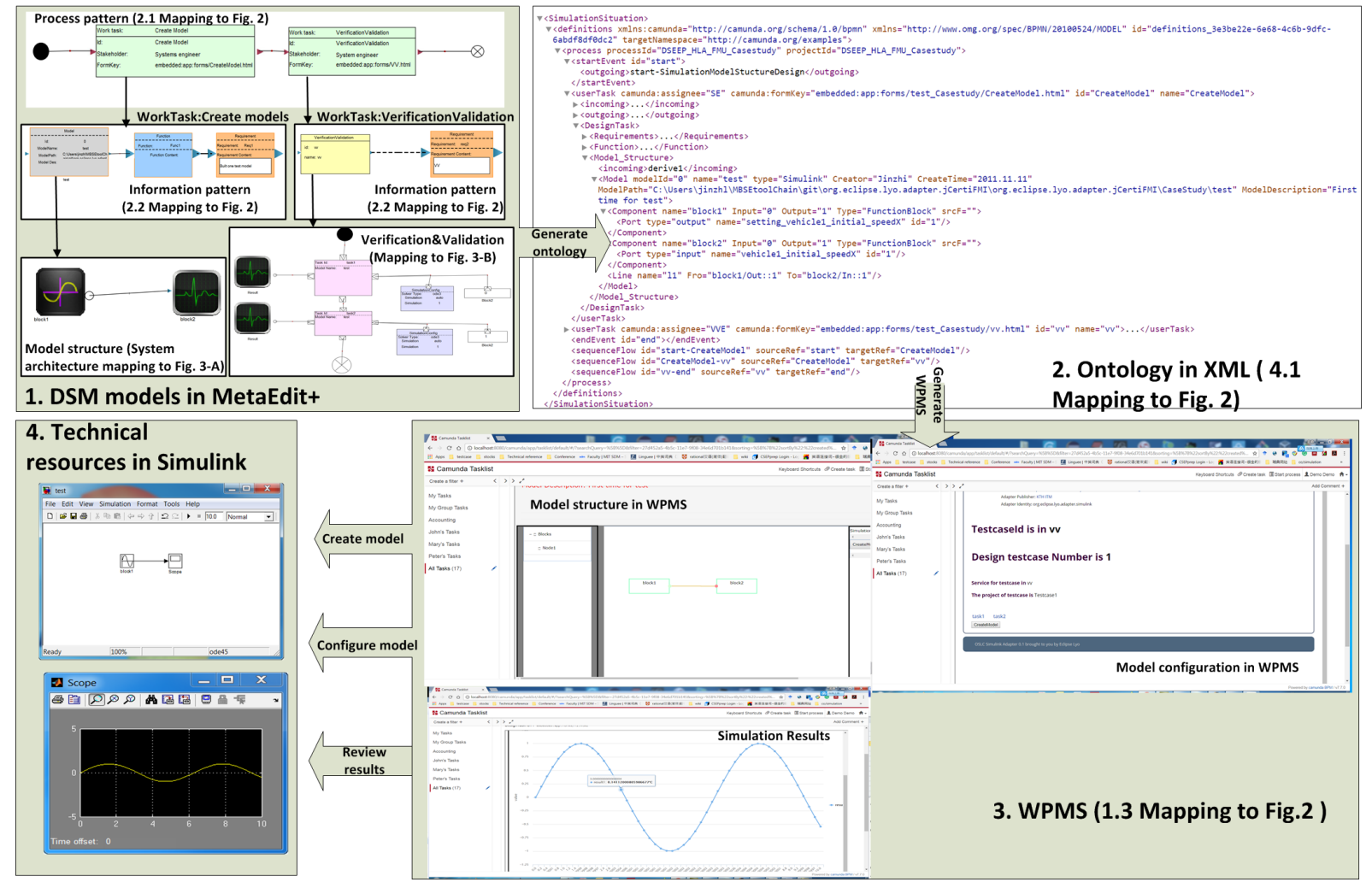

Fig. 5. Case Study

Through the service-oriented approach based on OSLC, technical resources - such as Simulink blocks, models and APIs - are wrapped into web-based services which promote their interoperability.

In our previous work, we have summarized two improvements of adopting a service-oriented approach [21]:

1 Improve Tool-integration: Using service-oriented approach, data and models in different tools are represented as RESTful services in order to be accessed by other tools. APIs in tools are represented as services which makes tools can be controlled in a unified platform. This leads to an improvement of tool interoperability and reusability.

2 Improve the "interrelationships" Management of technical resources: The services enables process integration between process management system and related technical resources which improves traceability and consistency between system development and implementations.

3 Practices of systems of systems (SOS). The SPIRIT framework is an exemplified SOS. Its related research provides engineers a industrial practice to understand SOS.

From our experiences, we note that there are still several limitations when the framework is used to implement the industrial practices for tool-chain development. Firstly, it is challenging to define a clear system boundary for the social network. Stakeholders should formalize their workflows (including development process and information) and define the related social networks based on systems engineering views. Secondly, tools for current CPS development are always specific and closed environments thus not enough APIs can be provided to support service development. Thirdly, interfaces between the MBSE tool-chain and existing PDM and PLM platforms should be defined. Open standards, such as OSLC can promote the interoperability between tool-chain and existing platforms.

From the technical view of the MBSE tool-chain, future work should be considered: DSM models can be formalized based on meta-models, however, except for the needs of domain-specific views, the meta-meta models (semantics and syntax) also need to be extended. Then further research would be done to improve ontology by considering open standards. Moreover, during service-orchestration, specifications of service (such as URI of each service) need to be further defined. In this paper, we adopted a sample case study to illustrate how the prototype supports Matlab/Simulink executing the simulations. Further V\&V techniques, such as co-simulation would be extended in the future.

\section{Conclusion}

In this paper, we proposed the SPIRIT framework to support MBSE tool-chain development. The framework integrates social networks of stakeholders, CPS development processes, system artifacts and technical factors, e.g. modeling methods and technical resources which can capture related requirements 
about the target tool-chain development. Moreover, based on the SPIRIT framework, a service-oriented approach is used for tool-chain development to promote interoperability. The tool-chain encompasses domain-specific modeling, process management based on IT platforms and standard based toolintegration. With the developed tool-chain prototype, CPS developers can formalize their products from a domain-specific view, deploy related technical resources with well-managed and good interoperability, and implement related verification $\&$ validation automatically.

\section{REFERENCES}

[1] M. Broy, M. Feilkas, M. Herrmannsdoerfer, S. Merenda, and D. Ratiu, "Seamless Model-Based Development: From Isolated Tools to Integrated Model Engineering Environments," Proceedings of the IEEE, vol. 98, no. 4, pp. 526-545, Apr 2010.

[2] J. Johnson, "The latest developments in design data exchange: towards fully integrated aerospace design environments," in Proceedings of 22nd International Congress of Aeronautical Sciences, Agu 2000.

[3] B. Beihoff, S. Friedenthal, D. Kemp, D. Nichols, C. Oster, C. Peredis, H. Stoewer, and J. Wade, "Systems Engineering Vision 2025," Tech. Rep., Mar 2010.

[4] J. Lu, D. Chen, D. Gürdür, and M. Törngren, "An Investigation of Functionalities of Future Tool-chain for Aerospace Industry," INCOSE International Symposium, vol. 27, pp. 1408-1422, Jul 2017.

[5] J. Fitzgerald, C. Gamble, P. G. Larsen, K. Pierce, and J. Woodcock, "Cyber-Physical Systems Design: Formal Foundations, Methods and Integrated Tool Chains," in 2015 IEEE/ACM 3rd FME Workshop on Formal Methods in Software Engineering, vol. 6396 LNCS, May 1990, pp. 40-46.

[6] E. A. Lee, "CPS Foundations," in Proceedings of the 47th Design Automation Conference on - DAC '10, New York, New York, USA, 2010, pp. 737-742.

[7] D. Verma and W. Fabrycky, "Systematically Identifying System Engineering Practices and Methods," IEEE Transactions on Aerospace and Electronic Systems, vol. 33, no. 2, pp. 587-595, Apr 1997.

[8] R. Mannadiar and H. Vangheluwe, "Domain-specific engineering of domain-specific languages," in Proceedings of the 10th Workshop on Domain-Specific Modeling. ACM, 2010, p. 11.

[9] H. Blom, D. Chen, H. Kaijser, H. Lönn, Y. Papadopoulos, M.-O. Reiser, R. T. Kolagari, and S. Tucci, "EAST-ADL: An Architecture Description Language for Automotive Software-intensive Systems in the Light of Recent use and Research," International Journal of System Dynamics Applications (IJSDA), vol. 5, no. 3, pp. 1-20, 2016.

[10] C. H. Roth Jr and L. K. John, Digital Systems Design Using VHDL. Cengage Learning, 2016.

[11] M. K. S. Biehl, "A Modeling Language for the Description and Development of Tool Chains for Embedded Systems," Ph.D. dissertation, 2013.

[12] Open Services for Lifecycle Collaboration, "Oslc primer," Tech. Rep., 2008.

[13] H. Broodney, U. Shani, and A. Sela, "Model Integration - Extracting Value from MBSE," INCOSE International Symposium, vol. 23, no. 1, pp. 1174-1186, Jun 2013.

[14] P. Braun, "Metamodel-based integration of tools," in Proceeding of ESEC/FSE. Citeseer, 2003.

[15] C. Hein, T. Ritter, and M. Wagner, "Model-driven tool integration with modelbus," in Workshop Future Trends of Model-Driven Development, 2009, pp. 50-52.

[16] W. Scheer, August and M. Nüttgens, "ARIS Architecture and Reference Models for Business Process Management," in LNCS, 2000, vol. 1806, pp. 376-389.

[17] M. Dumas and A. H. Ter Hofstede, "UML Activity Diagrams as a Workflow Specification Language," in International Conference on the Unified Modeling Language. Springer, 2001, pp. 76-90.

[18] R. Shapiro, S. White, N. Palmer, and et al., "BPMN 2.0 Handbook," Tech. Rep., 2010

[19] A. Fernandez, "Camunda BPM Platform Loan Assessment Process Lab," Tech. Rep.
[20] S. Kelly, K. Lyytinen, and M. Rossi, "Metaedit+ a Fully Configurable Multi-user and Multi-tool Case and Came Environment," in Advanced Information Systems Engineering. Springer, 1996, pp. 1-21.

[21] J. Lu, D. Gürdür, D.-J. Chen, J. Wang, and M. Törngren, "Empiricalevolution of frameworks supporting co-simulation tool-chain development," in Trends and Advances in Information Systems and Technologies, Á. Rocha, H. Adeli, L. P. Reis, and S. Costanzo, Eds. Cham: Springer International Publishing, 2018, pp. 813-828. 